\title{
Towards Simulating the Impact of National Culture on Organizations
}

\author{
Loïs Vanhée ${ }^{1,2}$, Frank Dignum ${ }^{2}$, Jacques Ferber ${ }^{1}$ \\ ${ }^{1}$ LIRMM, University of Montpellier II, France \\ ${ }^{2}$ Utrecht Universiteit, The Netherlands
}

\begin{abstract}
Both culture and organizations are concepts which have been partially formalized. Only some of their aspects have been specified to build agent-based models. In this conceptual article, we identify and characterize the features that should be considered when building an agent-based model of an organization taking into account the influence of culture. In particular, we investigate the impact culture can have on the delegation, coordination, control and normative structures of organizations and on the way these structures are used. Moreover, we describe how this cultural impact would influence the three central performance criteria of organizations: efficiency, flexibility and robustness.
\end{abstract}

Keywords: Simulating organizations, Simulating social complexity, Agent models of social behavior

\section{Introduction}

Why do some organizations succeed to expand their operations abroad while others fail? Many reasons are given. Often, individuals from the abroad part of the organization are declared responsible of this failure. They may constantly need to be directed in order to perform any action or they may take counterproductive initiatives. They may spend their time defining how things shall be done and do nothing or even overlook specifications and produce inappropriate results. Even worse, this distrust can be reciprocal and the abroad branch might also blame the parent one. The effects of such underlying expectations are embedded in culture. Organizations, as a means to create interaction amongst several individuals, are naturally sensitive to these expectations. Hofstede et al. [9] [10] studied and described the impact of culture on human behavior. They explained how copy-pasting the management style of one country can fail in another. Although this research is empirically grounded, it only gives a descriptive and intuitive account of the impact of cultures on organizations. In order to design, simulate and analyze models of organizations incorporating cultural influences, we must first formalize this intuitive description.

In this conceptual paper, we move one step forward the formalization of the impact of culture on an organization and on its performance. In particular, we focus on influence of culture from outside the organization, sometimes 
called national culture. This culture does not emerge from within the organization, which is referred as organizational culture [10] or corporate culture. To this extent, we link each aspect of organizations with each Hofstede's cultural dimension. In order to have a perspective that integrates each organizational aspect and each cultural dimension as wholes, we describe how each organizational aspect is influenced by culture but also how each cultural dimension influences the organization. Using these orthogonal descriptions, a modeler can build a model of culture and organizations which is coherent with both theories of culture and organizations. Moreover, the effect of culture on organizations influences the organizational behavior. Thus, the cultural effect can be observed from outside of the organization. In this article, we describe how culture would affect the performance criteria (efficiency, flexibility and robustness) of an organization. This description can be used by a simulator to determine if the shift of organizational performance due to a different cultural setting is conform to $a$ priori expectations.

We describe the relevant previous work about culture and organizations in Section 2, then we explain the impact of culture on organizations in Section 3. Finally, we present the consequences of this impact on the organization performance in Section 4.

\section{Previous work}

\subsection{Culture}

Culture can be defined as social knowledge. For instance Hofstede et al. [10] defines culture as values and practices (Figure 1). Values are a set (or an order) of dilemmas considered by individuals when interacting with the world (e.g. the "evil versus good" dilemma is more important than "rational versus irrational" one). Practices encompass rituals (e.g. saying "hello"), heroes and symbols. But, the particularity of cultural knowledge (especially of values) is that it is expected to be shared with other individuals. This expectation dramatically affects interactions, positively if they are shared and possibly negatively otherwise (why is the leader so bossy? Why do they lack so much subordination?).

Even if the concept of culture has not been formalized yet, Hofstede et al. [10] empirically classified cultures (independently of any representation) along 5 national dimensions: power distance (PDI), individualism (IDV), masculinity/femininity (MAS), uncertainty avoidance (UAI) and long-term orientation (LTO). Power distance influences the expectation and importance given to power statuses. Leaders are expected to take directions and subordinates to obey and not take initiatives. E.g.: China, Russia (high PDI) opposed to Scandinavian countries (low PDI). Individualism influences the definition of individual identity. The lower the IDV, the more one individual's identity is linked to his or her social context (e.g. relatives, colleagues). Thus, one's individual goals and actions (and the claim for this action) are more or less linked to him/herself or to his/her context. This context leads to a collective image that has to be preserved (helping each other, hiding errors, rejecting outsiders). Conversely, in high IDV 
cultures, individuals expect a treatment independent of any context. E.g.: USA, Great Britain (high IDV) opposed to South American countries (low IDV). Masculinity indicates preferences on assertiveness, toughness, focus on performance and material success. Good performance should be recognized and rewarded, leading to competition. Conversely, low MAS cultures favor modesty, tenderness and high quality of life. Interactions focus on building cooperation and establishing consensus. E.g.: Scandinavian countries (low MAS) versus Japan, Italy (high MAS). Uncertainty avoidance favors the desire for clear and explicit situations with predictable outcomes. This desire leads to establishment of rules (formal or not), making everything explicit with low ambiguity. Conversely, individuals with low UAI culture dislike the presence of rules. They tend to accept more easily situations with unspecified behavior or unclear outcome. E.g.: Greece, Japan (high UAI) versus Sweden, China (low UAI). Long-term orientation influences the time span considered when taking decisions. In high LTO culture, rewards can be sacrificed for better ones later, relationships are built on long-lasting trust and rules are flexible. Conversely, individuals in low LTO culture focus on immediate success, avoiding failure and decisions rely on dogmatic rules (e.g. total commitment, best profit commitment). E.g.: Extreme-Asian countries (high LTO) versus Canada, Great Britain (low LTO).

In computer science, culture has been investigated from two perspectives. The first one studies culture as a dynamic system of values (or memes) propagation. For instance, [2] studies the formation of cultural clusters or [4] the emergence of suboptimal equilibria. Nonetheless, these models consider only superficially the influence of culture on agent behavior. The second perspective focuses instead on the influence of culture on the decision making process, considering culture as a static parameter. For instance, Dignum [6] describes how cultural values can influence the plan selection process in considering the constraints imposed to the agent. In the same direction, Dechesne et al. [5] models the influence of culture on norm emergence. This model is used to investigate why the European smoking ban is being accepted in certain countries while not in others.

\subsection{Organizations}

Organizations are social structures created in order to accomplish a given goal. Morgenstern [15] describes organizations by introducing six key organizational features: goals, workflow, roles, structures (coordination, delegation, control and information), failures and norms.

An organization aims at achieving a goal, which is a set of tasks. Tasks are solved in executing unitary operations which may require some specific competence. The workflow [16] defines how these operations are linked to each other. Each operation is associated to a role possessing the right competences. These roles are connected with each other via the coordination structure, which should match the order of operations defined by the workflow. Individuals are allocated to roles and they are responsible for successfully performing the operations attached to their roles. Finally, a role can delegate an operation to another role if they are linked in the delegation structure. Delegation can be used to balance the 
workload and divide the work. The control structure determines roles in charge of monitoring operations of other roles. This information can be used to verify the success of an operation, the state of a task or to change the organization but also to prevent costly failures. Failures can be caused by operations incorrectly performed or tasks not handled by the workflow. In this case, exception handling rules can be used to find a solution to resolve these failures. Norms can be developed in order to avoid failures or to standardize processes of the organization. Finally, the information structure determines which information shall be transferred to whom and when. This structure encompasses other structures but can also be necessary for other purposes (e.g. storing information about the past failures). This information can then be used for longer-term learning. Organizational learning is a background process gathering past experiences of an organization in order to improve future performance.

Mintzberg [14] synthesized 5 frequent organizational patterns, each one fitting a particular purpose: simple structures, machine bureaucracies, professional bureaucracies, divisionalized forms and adhocracies. Simple structures are flat hierarchies composed of few leaders tightly supervising large groups of subordinates. This pattern is fit for simple tasks in dynamic environments (e.g. a grocery). Machine (or full [10]) bureaucracies are pyramidal hierarchies of specialists. Each branch is specialized in a domain, leaders encompass the specializations of their subordinates. This hierarchy centralizes all structures: leaders delegate, control and handle exceptions of their subordinates. Norms standardize the work processes, coordinating operators at the lowest level. This pattern fits repetitive tasks in simple and stable environments (e.g. mass production). Professional bureaucracies are composed of teams of autonomous experts. Training leads to a standardization of skills allowing complex sequences of operations to be performed while requiring minimal coordination. This pattern fits stable and complex environments (e.g. hospitals). Adhocracies are unstructured networks. Their structures are flat, thus anyone can coordinate, delegate or control anyone else. Individuals tend to cluster in working teams depending on the task to be solved. This structure is fit for complex and dynamic environments (e.g. software development). Finally, divisionalized forms are macro-organizations containing multiple sub-organizations. Each sub-organization (which tends to become a machine bureaucracy) aims at different markets. The key part of these organizations is the link between the top direction and each sub-organization. This pattern is more balanced and fit various environments.

Organizations have also been studied in the field of computer science. These studies have two main purposes: model organizations to build software [1] and study human organizations. This article focuses on the latter approach. [8] uses Morgenstern's description to model organizations. They are represented with a 3 layer directed multigraph. Vertexes represent the roles and the edges determine the delegation, coordination and control structures. The authors use this representation to build organizational performance measures and describe properties of the organizational behavior. [13] investigates organizational performance evaluation through simulations. Simulations are used in particular to evaluate 


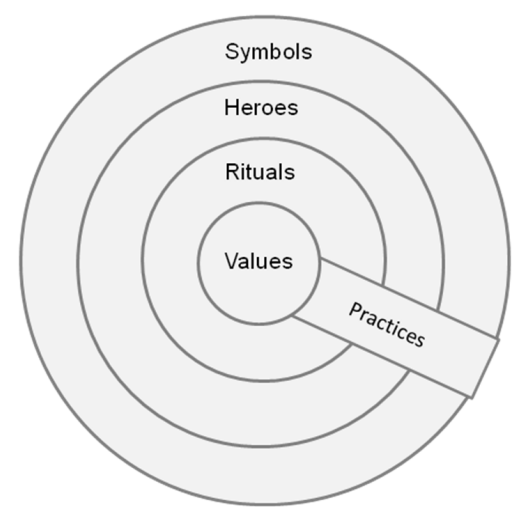

Fig. 1: Hofstede's model of cultures (onion diagram) [10]

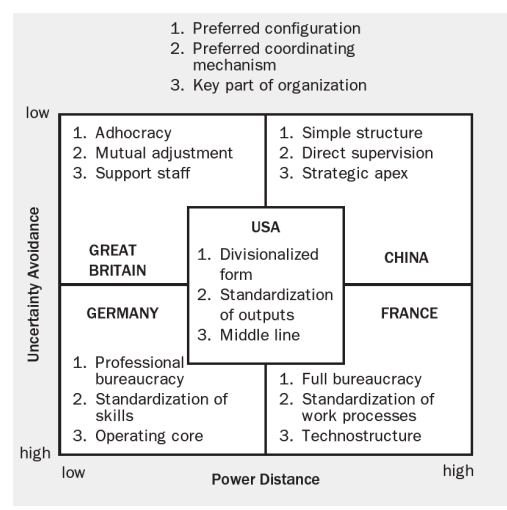

Fig. 2: Mintzberg's five preferred configurations of organizations, from [10]

the costs involved by congestion and evolving tasks. This evaluation of performance can be used to propose new organizational shapes. For instance, [11] uses genetic algorithms to optimize organizational design. Similarly, [3] presents an expert system proposing organizational change and supporting its propositions. The long-term goal of this article is to introduce the influence of culture in a model evaluating the performance and proposing adaptations for multi-cultural organizations.

\section{Culture and organizations}

Hofstede [10] links culture and organizations in two ways: the emergence of an organizational culture and the influence of national cultures on organizations. The first approach considers that organizations, as relatively closed interaction system, grow a culture. This culture is highly domain dependent: bank cultures are relatively similar but different from factory cultures. In this article, we focus instead on the second approach, which investigates why and how two organizations with similar purposes differ due to the influence of national cultures. Hofstede [10, ch.9] illustrates the Mintzberg type of organization that would emerge if organizations were only influenced by culture (Figure 2). In practice, this influence is not so extreme: even in a low UAI culture, hospitals require experts and a minimal amount of regulations. Nonetheless, culture can still influence some aspects of the organization (e.g. emphasize care vs efficiency). Similarly, a local sport club is more easily culturally driven than a hospital. Describing in detail the influence of each cultural dimension on each organizational feature would require a large amount of space. A summary of these links is presented in Table 1. Nonetheless, in order to build coherent models of culture and organizations, in the following sections we describe how each cultural dimension can influence organizations and how culture can influence each organizational feature. Note that cultural influence drives organizational behavior, but actual implementation also depends on other parameters like the environment. 


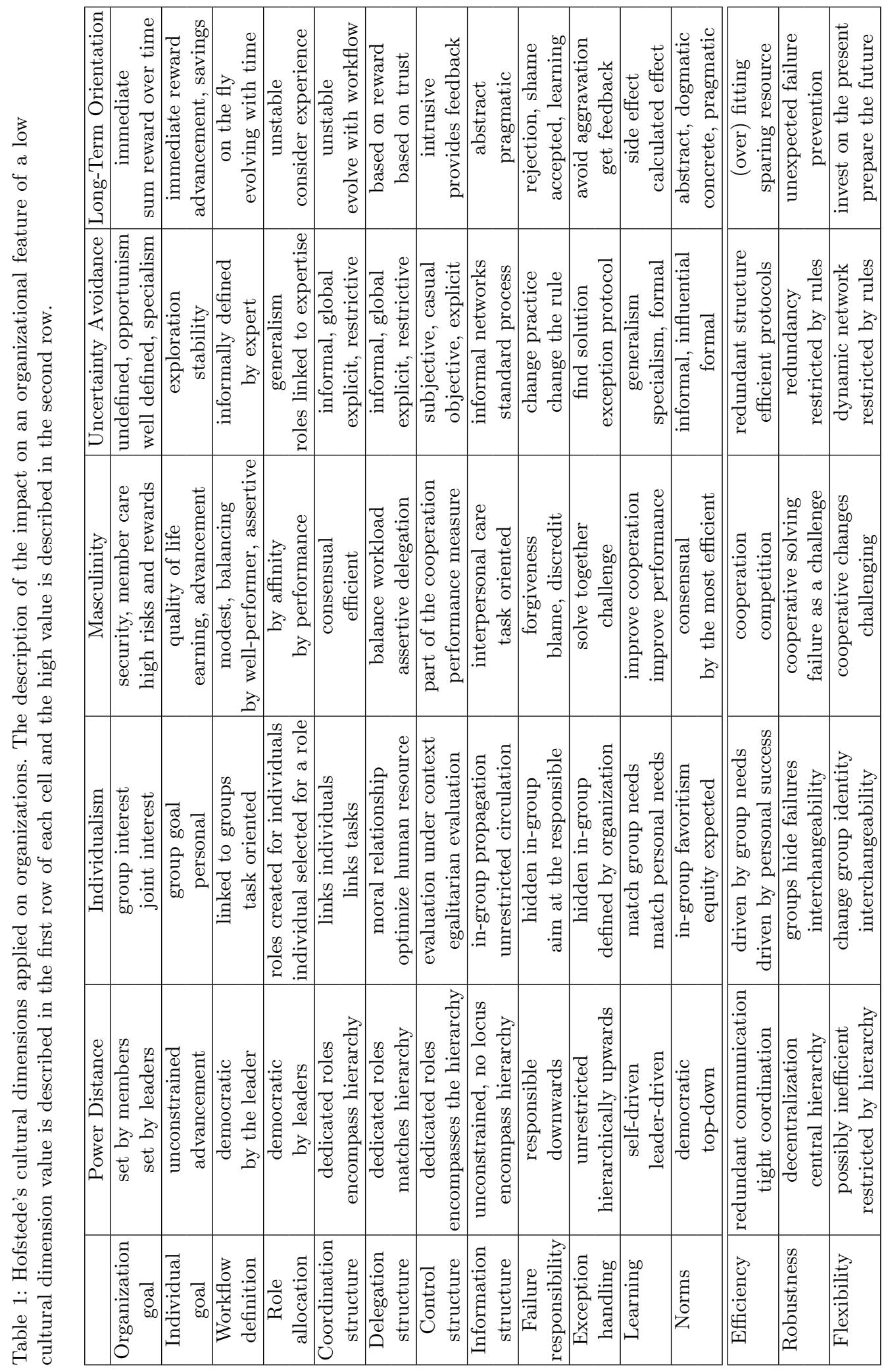




\subsection{The impact of each cultural dimension on organizations}

Power Distance: PDI influences the perception of power associated with individuals. Power, in general, is the capability of an individual to influence or restrict the behavior of another individual (e.g. affecting his/her welfare). In high PDI cultures, individuals expect to be lead by one unique individual while in low PDI cultures, power is expected to be spread. The organizational context is particularly favorable to the creation of formal and informal power. Formal power arises mainly through organizational structures and the possibility to establish and apply norms. Informal power results from the praise given to an individual (high MAS), informal networks (low IDV), and/or expertise (high UAI). In high PDI cultures, individuals want the power to be concentrated in few hands, leading to a fusion of structures. Leader uniqueness stresses the structural shapes towards trees. In low PDI, individuals prefer decentralized power. So, structural merging is avoided, leading to more balanced networks. PDI also influences the importance given to individual status. Thus, in high PDI cultures, a leader's opinion and welfare are more valuable than those of the subordinates, which impacts decision making processes (e.g. autocratic delegation).

Individualism: IDV influences the importance given to one individual's context. This context can introduce important side-effects on organizations. Since the identity of an individual is linked to his/her context, individuals sharing a context tend to protect each other and reject outsiders. Thus, the notion of fairness depends on IDV: in low IDV cultures, in-group favoritism is expected while in high IDV ones equality is expected. IDV influences individuals goals, towards personal or context interest. The responsibility for a failure charged on an individual or on his/her context depends on IDV. To this extent, in low IDV cultures, a failure notification from an out-grouper is more troublesome than from an in-grouper.

Masculinity: MAS influences the preference towards performance and success over care and stability. Thus, organizations in high MAS cultures prefer assertive goals (even if potentially risky or unachievable) and members, stressing on individual performance. Conversely, low MAS organizations favor modest and achievable goals keeping their members satisfied. MAS also influences the workflow and the role allocation. The stress on performance impacts in turn the tendency towards competition (high MAS) or cooperation (low MAS). Moreover, MAS influences the importance given to performance measure (carried by the control structure), success recognition and failures. In high MAS cultures, failures display discredit the individual (potentially leading in turn to retrogradation or removal) while in low MAS settings, failures can be forgiven in order to pursue cooperation.

Uncertainty Avoidance: UAI influences the need of well defined and predictable situations. In organizations, this need impacts the accuracy of the process definition. Thus, workflow, roles (through expertise), processes (operations to be performed, how the task flows in the organizational structure), operation outcomes and norms are more explicit. In high UAI cultures, processes strictly follow the predefined structures and a regular control validates the completion 
of a task. Conversely, in low UAI cultures, structures are less formally defined, paths taken by tasks are less predictable and control is more rare. This lack of formalism can be represented with an organic informal structure.

Long Term Orientation: LTO influences the importance of immediate rewards and lack of failures versus investments leading to greater expected benefits in the future (e.g. training individuals, updating machines). Thus, low LTO organizations tend to dramatically change their activity to any more profitable one while high LTO organizations update their strategy more smoothly. LTO influences the revision of rules for decision: from dogmatic commitment to rules versus pragmatic rules evolving with time and experience. This choice influences the dynamism of structures and norms. LTO also influences the importance of trust in interactions. In low LTO cultures, these interactions are more efficient but also more risk-prone (e.g. members can more easily quit).

\subsection{The impact of culture on organizational aspects}

Structures Organizational structures (coordination, delegation, control, information) link individuals with each other. Structures can be merged and centralized in a single hierarchy of power (high PDI). This hierarchy determines leader/subordinate relationships where leaders instruct, help and verify subordinates operations. Conversely, power can also be spread in organization with low fusion and no hierarchical distribution of structures (low PDI). Thus, an individual can have several leaders (e.g. in a matrix structure) or two different roles can manage the delegation and the control of a third one. In extreme cases, organizations can be represented by a complete graph (e.g. adhocracies). Thus, for instance individuals may delegate tasks to anyone else. In addition, the importance given to expertise (influenced by UAI) also influences the shape of the organization. Expertise can be important for role creation and to link individuals (high UAI). Then, the organization tends to be structured in teams (low PDI) or hierarchies of experts (high PDI, where leaders encompass their subordinates expertise). Otherwise, links are less restricted by formal structures, leading to unrestricted teams (low PDI) or hierarchies of power (high PDI). Figure 2 links this description with Mintzberg's synthetic organizations. Finally, note that organizations can be attached to their formal structures and experts (high UAI) making individuals rely explicitly on these structure to handle tasks, or they can rely on informal structure emergence (low UAI).

Organizational structures can evolve with time. Goals can change, leading to changes in workflow and thus in structure. But, roles in charge of managing organizational change differ depending on the organization and culture. Adding or removing new members can be done by a leader (high PDI), influenced by experts (high UAI) or the opinion of best performers (high MAS). Otherwise, this process can be more democratic and consensual. The reasons for hiring or firing employees are also culturally driven: by expertise (UAI), by apparent efficiency (MAS), by familial background (IDV) and the way to manage the organizational agenda (LTO). 
Moreover, culture also influences how interactions are carried out through these structures. Taking the decision to interact with someone else is dependent on culture. This decision can be made explicit and objective through procedures (UAI, e.g. a procedure describes to control one item for every 1000). Status differences can influence the decision to initiate it (PDI e.g. an individual may prefer to avoid using delegation links towards individuals with higher status). Individuals can care or not about the state of mind of the other one (MAS, e.g. an individual may prefer to avoid delegating a task to an individual with personal problems in low MAS. Contrarily, demands can be voluntarily set above the capability of the receiver in order to force him/her to outperform in high MAS). Individuals may expect a degree of fairness (e.g. balance the ungratifying work) or accept different treatment due to context (IDV). Finally, trust and longer-term goals can be taken into consideration when initiating an interaction (LTO, e.g. always delegate tasks to the most profitable individual or delegate simple tasks to newcomers who need experience).

The protocols underlying interactions are also culturally driven. In some cultures, the subordinates expect to receive instructions before acting while in others they expect a degree of autonomy (PDI). Similarly, communications can be more assertiveness or consensual and explanatory (MAS). Thus, the balance between proposing and imposing is dependent on PDI and MAS. PDI influences the acceptance of decision from higher power individuals, while MAS influences the care given to other's desires in decision. Thus, for instance, in a high PDI, low MAS organization, a leader can delegate a task if he/she considers that the subordinate is willing to do it. In addition, the UAI influences the amount of standardization of the interactions (e.g. standardized delegation messages). Finally, the amount of context in the message is influenced by the IDV.

Failures Defining a failure and deciding how to handle it are also culturally dependent. Some organizations can recognize as a failure a document wrongly filled in while others are not worried unless a factory is destroyed overnight. The occurrence of a failure is linked to the detail given to the processes and its expected outcome (UAI). Moreover, an organization which performs intensive control is more sensitive to failures (UAI, PDI). The consequence is that more failures are reported but their gravity is generally lower.

When a failure is observed, it may be fixed with a failure handling mechanism. Organizations centralized in a hierarchy tend to transfer the failure upwards and delegate it to the correct service (high PDI). A standard failure handling mechanism can be designed (high UAI) or it is solved by hand with the informal network (low UAI) to handle it. The group where this failure occurred can try to solve it locally (IDV). Some individuals may look forward to failures to gain recognition by solving them or lose this recognition and solve them through cooperation (MAS). Finally, the behavior when faced to error handling can vary, like trying to resolve it and forget about it or trying to get more feedback about its origin but possibly without any additional reward (LTO).

When a failure occurs, for various reasons organizations may want to determine a responsible. The failing individual can be classified as inefficient (high 
MAS), unfit for his/her position (high PDI) and endangering the group (low IDV). Some tolerance can be accepted to avoid hurting the faulty individual (low MAS) or because failure is part of a learning process (high LTO). The blame can be determined by explicit and objective rules (UAI), or by a special role, like a leader (PDI) or a special control institution (UAI). Individuals can expect more or less fairness from the system with regard to their context (IDV). The context itself may be in charge of blaming the faulty individual. Leaders can try to reject the responsibility in order to avoid being discredited (PDI).

Norms Norms are rules restricting the individual's behavior, but they can nonetheless be violated in exceptional cases. In an organization, norms can serve to improve performance or avoid failures (e.g. a vaccine should be kept below $15^{\circ} \mathrm{C}$ ). The category of individuals determining the norm is influenced by culture. As for decision making, this individual can be a leader (PDI), an expert (UAI) or the best performer (MAS). These norms can be more or less democratic and consensual (PDI, UAI, MAS). The definition and the content of the norm are also influenced by culture. Rules can be more or less explicit and objective (UAI) and they can be connected to various features of the organization, like expertise (UAI) and position (PDI, MAS). Finally, the individuals in charge of applying the norm can vary. This individual can be a leader (PDI), a special role (UAI), the best performer (MAS) or any colleague (low PDI, low UAI). Some organizations can more easily forgive violations than others (LTO, MAS). The importance of blame can also vary (MAS, PDI). Individuals can also expect a relative fairness or group favoritism when being judged (IDV).

\section{Consequences of culture on organizational performance}

Efficiency measures the costs to achieve a task. In multi-agent and multi-task goals, the cost can be defined in various ways (e.g. individuals can cost over time or by operation). The number of messages exchanged in order to achieve a task is a generally reliable indicator for efficiency. Efficiency is dependent on the task complexity and the variability of the task space (see Figure 2).

Simple tasks have low coordination costs and predictable operations. A strong leader (high PDI), as a central node, can optimize coordination while keeping inter-individual communication low. Conversely, complex tasks require better competence and communication costs are higher. In this case, efficiency is gained by giving more autonomy to individuals (low PDI). Static tasks are efficiently handled via standardization (being a formal work process or a formal training), leading to specialization (high UAI). Contrarily, dynamic tasks require that individuals are not bound to rules (low UAI). IDV has a positive effect on efficiency: high IDV cultures make individuals responsible for their operations. In low IDV cultures individuals accomplishment is blurred by the group, reducing incentives to perform well. The impact of MAS on efficiency is mitigated: the desire of success is a motive which may also create inefficient competition.

[7] proposes a twofold approach to robustness : congestion and connectivity. Congestion robustness measures the organizational capability to cope with 
concurrent tasks, highlighting bottlenecks. Connectivity robustness measures the organizational capability to cope with the disappearance of some members (e.g. sick leave). For both approaches, robustness increases when a task can be performed through multiple paths and actors which implies the creation of redundant roles and links.

PDI has a negative impact on this redundancy: the higher the PDI, the more individuals prefer to obey a single leader, driving the organization to a tree-like structure without redundancy. Similarly, UAI influences the use of explicit links: in case of failure, alternate routes must have been conceived beforehand. MAS has a mitigated influence on robustness. In high MAS cultures, individuals expect recognition when doing extra work when the organization has a problem while in low MAS ones, individuals want to cooperate and protect the organization even at the cost of extra work. LTO has a positive effect: organizations with high LTO culture tend to pragmatically consider failures and establish appropriate prevention and learn from past failures.

Flexibility measures the range of tasks that can be coped with by the organization. Thus, organizations with individuals that are generalist and not bound to formal structures (low UAI) are naturally more flexible. Similarly, PDI prevents the task to flow without validation of the leader which may lead to excessive costs. The assertiveness and competition driven by high MAS cultures have a positive influence on flexibility. Low MAS cultures are more conservative and less risk-taking. LTO has mitigated influence on flexibility: high LTO organizations are prone to early and smoothly shift their activity to increase their long-term profit while low LTO organizations can sharply change their activity as soon as the new goal is more profitable.

\section{Conclusion and future work}

In this conceptual article we highlight how cultural backgrounds influence structures and interactions occurring in an organization. We describe how cultural dimensions influence organizations and how organizational features are influenced by culture, emphasizing the individual level. Moreover, we present the consequences of cultures on organizational performance (efficiency, robustness, flexibility). This way, a modeler can use our work as a guideline to integrate culture and organizations in a single agent-based model. This model can be used to simulate organizational operations in various or multiple cultural settings, which can provide data about the cultural influence on organizational performance.

In the future, we plan to use this conceptual work as a reference point to build agent-based models of culture and organizations. In particular, we are interested in reproducing phenomena occurring in multi-cultural organizations or cross-cultural replication of organizational structures. For instance, we expect to observe an increase of inter-individual clashes due to cultural mismatch or inefficiency due to a misuse of an organizational structure. This work, in a longer term, aims at helping decisions makers in providing them deeper insight into how culture can influence the way their decisions will be interpreted and applied. 


\section{Acknowledgments}

The first author wishes to thank Gert Jan Hofstede for his fruitful interaction and Melania Borit for her feedback while writing this paper.

\section{References}

1. E. Argente, V. Julian, and V. Botti. Multi-Agent System Development Based on Organizations. Electronic Notes in Theoretical Computer Science, 2006.

2. R. Axelrod. The Dissemination of Culture: A Model with Local Convergence and Global Polarization. Journal of Conflict Resolution, 41(2):203-226, 1997.

3. Baligh, Burton, and Obel. Validating an expert system that designs organizations. Computational Organization Theory, pages 179-193, 1994.

4. S. Bura. Minimeme: Of Life and Death in the Noosphere. In From Animals to Animats 3 Proceedings of the Third International Conference on Simulation of Adaptive Behavior, pages 479-486. London: MIT Press., 1994.

5. F. Dechesne, G. D. Tosto, V. Dignum, and F. Dignum. No smoking here: values, norms and culture in multi-agent systems. In Artificial Intelligence and Law, 2012.

6. F. Dignum and V. Dignum. Emergence and enforcement of social behavior. In 18th World IMACS Congress and MODSIM09 International Congress on Modelling and Simulation., 2009.

7. P. S. Dodds, D. J. Watts, and C. F. Sabel. Information exchange and the robustness of organizational networks. Proceedings of the National Academy of Sciences of the United States of America, 100(21):12516-21, Oct. 2003.

8. D. Grossi and F. Dignum. Structural Aspects of Organizations. In Handbook of Research on Multi-Agent Systems: Semantics and Dynamics of Organizational Models, pages 190-219. 2009.

9. G. Hofstede. Motivation, leadership, and organization: Do American theories apply abroad? Organizational Dynamics, 9(1):42-63, 1980.

10. G. Hofstede, G. J. Hofstede, and M. Minkov. Cultures and Organizations: Software of the Mind, Third Edition. 2010.

11. Kevin Crowston. Evolving Novel Organizational Forms. Computational Organization Theory, pages 19-38, 1996.

12. M. Lamieri and D. Mangalagiu. Interactions Between Formal and Informal Organizational Networks. In Handbook of Research on Multi-Agent Systems: Semantics and Dynamics of Organizational Models. 2009.

13. R. E. Levitt, G. P. Cohen, J. C. Kunz, C. I. Nass, T. Christiansen, and Y. Jin. The "Virtual Design Team": Simulating How Organization Structure and Information Processing Tools Affect Team Performance. In Computational Organization Theory. 1994.

14. H. Mintzberg. Structure in 5's: A Synthesis of the Research on Organization Design. Management Science, 26(3):322-341, 1980.

15. O. Morgenstern. Prolegomena to a theory of organization. Technical report, 1951.

16. W. M. P. Van Der Aalst, A. H. M. Ter Hofstede, B. Kiepuszewski, and A. P. Barros. Workflow Patterns. Distributed and Parallel Databases, 14(1):5-51, 2003. 